\title{
Study of atomic and condensed atomic indices for reactive sites of molecules
}

\author{
P KOLANDAIVEL*, G PRAVEENA and P SELVARENGAN \\ Department of Physics, Bharathiar University, Coimbatore 641 046, India \\ e-mail: ponkvel@hotmail.com
}

\begin{abstract}
In this paper, we have introduced the atomic descriptors $s(f)_{k}$ to determine the local reactive sites of the molecular systems during electrophilic, nucleophilic and radical attacks. The condensed Fukui function and the newly introduced condensed atomic descriptor have been calculated for six different systems, namely glycine, alanine, aniline, $\mathrm{BH}_{2} \mathrm{Cl}$, trans-FC(O)OF and $m$-anisidine. The individual atomic charges (gross charge) calculated by the MPA scheme have been used to calculate the condensed Fukui functions $\left(f_{k}\right)$ and the newly derived condensed atomic descriptors $(s f)_{k}^{\alpha}$ at B1-DZP level of theory. We carried out the calculation using the "stockholders" charge partitioning technique (i.e., Hirshfeld population scheme). The newly derived quantity gives the same reactive sites as the condensed Fukui functions, and the complexities associated with the negative Fukui functions are removed.
\end{abstract}

Keywords. Condensed Fukui functions; atomic descriptors; condensed atomic descriptors; electrophilic; nucleophilic; radical attacks.

\section{Introduction}

Density functional theory (DFT) ${ }^{1}$ is one of the important tools of quantum chemistry to understand the popular chemical concepts like electronegativity and chemical potential, ${ }^{2}$ ionization potential and electron affinity ${ }^{3}$ etc. The electron density based local reactivity descriptors; local hardness $\eta(r),{ }^{4}$ local softness $s(r)$ and the Fukui function $f(r)^{5}$ were proposed to explain the chemical selectivity or reactivity at a particular site of a chemical system. Recently, Geerlings et $a l^{6}$ showed that these local descriptors of DFT are essentially important in searching the "similarity of reactivity" of a group of molecules or atoms that are similar (in their structure and by extension in their electron density distribution). Electron density $\rho(r)$ is a property that contains all of the information about the molecular system and plays an important role in calculating almost all these chemical quantities. It has also been shown that local hardness $\eta(r)$ is a reliable intermolecular reactivity descriptor, ${ }^{7,8}$ and the local softness $s(r)$ and Fukui function $f(r)$ are more reliable intramolecular site selectivity descriptors. ${ }^{9,10}$ Several attempts have been made to evaluate the Fukui function (FF) indices. Parr and Yang ${ }^{11}$ proposed a finite difference (FD) approach to calculate FF indices i.e., nucleophilic, electrophilic, and

*For correspondence radical attacks. Further, number of methods and algorithms have been developed based on the partition of $\rho(r)$ on different atoms to define Fukui functions condensed on certain atomic regions. Yang and Mortier ${ }^{12}$ proposed three different condensed forms of $f(r)$, based on atomic charges of $N, N+1$, and $N-1$ electron systems. Nalewajski ${ }^{13}$ has studied the $f(r)$ indices in respect of atom-in-molecules (AIM). Cioslowski et al $^{14}$ have developed an FD approach based on spin-polarized method to calculate the Fukui function indices in the framework of the AIM method of Bader. ${ }^{15}$ The atomic and group resolution of $f(r)$ indices based on semiempirical method was proposed by Komorowski et $a l^{16}$. Although these indices are successful in generating the experimentally observed intramolecular reactivity trends in many systems, ${ }^{8,9}$ the occurrence of negative Fukui function has remained a puzzle for a long time. Senthilkumar et $a l^{17}$ have shown the existence of negative Fukui functions in a few triatomic molecules, where they discussed the correlation between effective hardness and condensed Fukui function using AIM, ab initio and DFT methods. In order to solve the negative Fukui function problem, different attempts have been made by various groups. ${ }^{18}$ Two different local reactivity descriptors, "relative nucleophilicity" and "relative electrophilicity", introduced by Roy et al ${ }^{19}$ based on FF indices solved the above problem. Roy et al ${ }^{19,20}$ have discussed several reasons for the appearance of negative 
FF values. One of the reasons, they have argued, is that according to the analytic definition of $\mathrm{FF}$ it is valid only when $\Delta N \rightarrow 0$. In that case, the fluctuation in the electron density distribution is minimal due to negligible relaxation in the corresponding cation and anion. However, in the definition of condensed FF's or local softness calculated by the finite difference method, we allow a change of the electron number by a large quantity, i.e., 1 and as a result there is a significant change in the electron-density distribution due to large relaxation in the corresponding cation and anion. This may cause depletion/accumulation of electron density on a particular site even though the global electron number is increased/decreased by 1. Another probable reason for the occurrence of negative FF indices might be the partitioning scheme, which we adopt to evaluate the condensed FF values. The well-known population schemes, Mulliken population analysis (MPA), ${ }^{21}$ natural population analysis (NPA) ${ }^{22}$ and molecular electrostatic potential (MESP) are found to produce negative FF values due to the inappropriate partitioning of charges. Roy et al ${ }^{19}$ proposed that the use of the stockholders' partitioning technique (Hirshfeld Population Analysis) developed by Hirshfeld ${ }^{23}$ provides non-negative Fukui function values. The basic idea of this scheme is that the partitioning of electron density to different atoms is fixed for $N, N+1$ and $N-1$ electron systems. The use of constant ratio ("sharing function") in $N, N+1$ and $N-1$ electron systems is the probable reason behind the HPA producing reliable positive FF values where in other popularly known schemes, this partitioning is improper. Chattaraj et $a l^{24}$ have proposed a "local philicity index" by generalizing both local and global electrophilicity as well as nucleophilicity, from the identity associated with the normalization of the Fukui function. Since the local philicity is a product of the FF and the global electrophilicity, it takes care of the local intramolecular (unlike FF) reactivity and site selectivity and the philicity is found to be positive everywhere. In this paper, we have established a new descriptor called "atomic descriptor" that greatly increases our understanding of the atomic sites. This newly introduced atomic descriptor not only contains profound information regarding electrophilic/nucleophilic local sites of a given molecule, but also removes the complexity associated with negative Fukui functions $f(r)$. A special treatment of the condensed atomic descriptors has been made on some small biomolecules such as glycine and alanine and some organic molecules such as aniline,
$\mathrm{BH}_{2} \mathrm{Cl}$, trans-FC(O)OF and $m$-anisidine. Due to the inherent nature of the population schemes, an attempt has been made to compare the reactive atomic sites calculated in a molecule by $f_{k}$ values with that of the sites obtained by the new descriptor.

\section{Theoretical aspects}

\subsection{Local reactivity descriptors}

The Fukui function proposed by Parr and Yang ${ }^{11}$ is one of the local reactivity indices that represent the system's response to a simultaneous perturbation in the total number of electrons $N$ and the external potential $\mathrm{V}(r)$ due to the compensating positive (nuclear) charges in the system. Fukui function $f(r)$ is defined as,

$$
f(r)=(\delta \mu / \delta \mathrm{v}(r))_{N}=(\partial \rho(r) / \partial N)_{\mathrm{v}(r) .}
$$

The local softness, which describes the response of any particular site of a chemical species (in terms of a change in electron density $\rho(r)$ to any global change in its chemical potential), is given by

$$
s(r)=(\partial \rho(r) / \partial \mu)_{\Downarrow(r) .}
$$

where $\rho(r)$ is the electron density at the site $r$, and $\mu$ is the chemical potential which is the negative of the electronegativity. ${ }^{2}$

The parameter $s(r)$ obeys the condition,

$$
\int s(r) \mathrm{d} r=S,
$$

where $S$ is the global softness which is defined as

$$
S=1 / 2 \eta=(\partial N / \partial \mu)_{\vee(r) .} .
$$

Rewriting (3) and then combining (1), (2) and (4) we have,

$$
s(r)=\left(\frac{\partial \rho(r)}{\partial N}\right)_{V(r)}\left(\frac{\partial N}{\partial \mu}\right)_{v(r)}=f(r) S=\left(\frac{\partial \mu}{\partial \vee(r)}\right)_{N} S,
$$

where $f(r)$ is the Fukui function introduced by Parr and Yang. ${ }^{11}$ Thus it is clear from (5) $f(r)$ can be used to study the relative site selectivity in a molecule (intramolecular reactivity sequence) where as $s(r)$ which contains global softness, in its definition, 
can be used as an indicator when comparing the reactivity of different molecules with respect to a common reaction partner.

The local softness condensed to an atom site say $k$, can be written as,

$$
s_{k}^{\alpha}=f_{k}^{\alpha} S .
$$

The Fukui function is an index of considerable importance for understanding the molecular behaviour of the species (neutral, monopositive and mononegative ions) understudy. The evaluation of the Fukui function values is not straightforward due to the difficulties associated with solving the derivatives given in (1). Yang and Mortier ${ }^{12}$ have given a simple procedure to calculate the atomic condensed Fukui function indices based on Mulliken population analysis (MPA) and on three possible forward, backward, and central finite difference approximations to the derivatives of (1), and can be written as,

$$
\begin{aligned}
f_{k}^{-}(r)= & q_{k}(N)-q_{k}(N-1), \\
& \text { governing electrophilic attack, } \\
f_{k}^{+}(r)= & q_{k}(N+1)-q_{k}(N), \\
& \text { governing nucleophilic attack, } \\
f_{k}^{0}(r)= & \frac{1}{2}\left(q_{k}(N+1)-q_{k}(N-1)\right), \\
& \text { governing radical attack, }
\end{aligned}
$$

where $f_{k}^{-}$and $f_{k}^{+}$describe the ability of an atom to accommodate an extra electron or to cope with the loss of an electron and $f_{k}^{0}$ is then considered an indicator for radical reactivity.

The behaviour of molecules as electrophiles/ nucleophiles during reaction depends on the local behaviour of molecules, i.e. on how the atomic sites of the molecule react towards the approaching reagent (electrophile/nucleophile/radical). So it is very important to infer the reactivity trends of local site during electrophilic or nucleophilic attack. As we know well, the intra molecular reactivity can be explained by either $s(r)$ or $f(r)$. In the present study, we pooled the local reactivity $f(r)$ and the local site selectivity $s(r)$ given in (6) and (7), as follows.

Considering the identity that $f_{k} / f_{k}=1$, in (6), we have,

$$
s_{k}^{\alpha}=f_{k}^{\alpha} S \cdot\left(f_{k}^{\alpha} / f_{k}^{\alpha}\right),
$$

$$
(s f)_{k}=\left(f_{k}\right)^{2} S \text {. }
$$

It may be noted that in (6) the function "atomic descriptor" $(s f)_{k}$, contains information on both $s_{k}$ and $f_{k}$. It takes care of both the local reactivity and selectivity of a molecule. This parameter allows one to infer the behaviour of local sites during electrophilic, nucleophilic and radical attacks since it is the combination of two local descriptors $s_{k}$ and $f_{k}$ that concerns more about selectivity and reactivity of the atomic species in addition to the global function $S$.

Three different types of condensed atomic descriptors $(s f)_{k}^{\alpha}$ can be readily obtained as,

$$
(s f)_{k}^{\alpha}=\left(f_{k}^{\alpha}\right)^{2} S,
$$

where $\alpha=+/-/ 0$ refer to nucleophilic, electrophilic and radical attacks, respectively.

i.e.

$$
\begin{aligned}
s_{k}^{+} f_{k}^{+}= & \left(f_{k}^{+}\right)^{2} S, \\
& \text { governing electrophilic attack, }
\end{aligned}
$$

$$
s_{k}^{-} f_{k}^{-}=\left(f_{k}^{-}\right)^{2} S,
$$$$
\text { governing nucleophilic attack, }
$$

$$
s_{k}^{0} f_{k}^{0}=\left(f_{k}^{0}\right)^{2} S, \quad \text { governing radical attack, }
$$

where (10a) predicts that the most electrophilic site in a molecule is the one which has the maximum $s_{k}^{+} f_{k}^{+}$value, which in turn is the softest region in a molecule.

\section{Computational details}

In the present study, we have chosen the low energy structures of two amino acids glycine and alanine ${ }^{25,26}$ and some organic molecules such as aniline, $\mathrm{BH}_{2} \mathrm{Cl}$, trans- $\mathrm{FC}(\mathrm{O}) \mathrm{OF}$ and $m$-anisidine to examine their preferred reactive sites in the gas phase and to examine the reliability of the newly described atomic descriptor. Since DFT method has provided comparatively good results on FF values ${ }^{27}$ we have chosen gradient corrected BLYP with LDA VWN correlation potential method for the computations. All the structures have been optimized at the gradient corrected BLYP method, with LDA VWN ${ }^{28}$ correlation functionals (we represent it as B1). The Huzinaga double-zeta basis set $^{29}$ with polarization 
(DZP) is used. For the above theory a vibrational analysis was performed for each optimized structures and in all the cases, it was verified that all the vibrational frequencies were positive indicating that all the structures were an energy minimum. Single point energy calculations have been performed at the above level of theory for the anions and cations of the conformers using the ground state optimized structure with doublet multiplicity. The individual atomic charges (gross charge) calculated by MPA have been used to calculate the Condensed Fukui functions $\left(f_{k}\right)$ and the newly derived descriptors $(s f)_{k}$ at B1-DZP level of theory using Amsterdam Density Functional program package (ADF). ${ }^{30}$ In order to check the reliability of Stockholders' charge partitioning technique i.e., Hirshfeld population analysis over MPA, the reactivity descriptors have also been calculated by the gross charges of HPA at B1/DZP level of theory.

\section{Results and discussion}

Tables 1 to 6 show the $f_{k}$ and $(s f)_{k}$ values for the molecules, glycine, alanine, aniline, $\mathrm{BH}_{2} \mathrm{Cl}$, trans$\mathrm{FC}(\mathrm{O}) \mathrm{OF}$ and $m$-anisidine calculated by MPA and HPA gross charges at B1/DZP level of theory. The optimized structures are shown in figure 1 in which the numbering of all the atoms is depicted.

From the tables one can find that the complexities associated with $f_{k}$ values due to the negative values being removed in the $(s f)_{k}$ values. In order to confirm that the atomic descriptor would produce the reactive sites without disturbing the trend, we have performed the calculation for the reactive sites of the stable structures of the glycine and alanine systems. The basicity of amines has been a challenge for the DFT descriptors of reactivity. Moreover, the basicity of amines can be studied by looking at the nucleophilic sites calculated by FF values. Theoretical calculations on some organic molecules including glycine and alanine has been carried out by Patricia Pérez et $a l^{31}$ to study reactivity, selectivity and site activation sites. In their work, they have concluded that the $\mathrm{N}$ atom of the amino group is the preferred site for protonation. The explanation in favor of $\mathrm{N}_{3}$ to be the most favourable reactive site for protonation is that (i) the hydrogen atoms of $\mathrm{N}_{3}$ are involved in intra-molecular hydrogen bonding with $\mathrm{O}_{5}$, which is doubly bonded with $\mathrm{C}_{1}$, and also (ii) the oxygen atom $\mathrm{O}_{5}$ pulls the electron density from the $\mathrm{C}_{1}$ carbon atom due to its higher electronegativity and consequently partial charges develop on these sites $\left(\mathrm{O}_{5}\right.$ and $\left.\mathrm{C}_{1}\right)$, leaving the nitrogen atom $\mathrm{N}_{3}$ the electron-rich site for protonation. The calculated condensed FF and $(s f)_{k}$ values of glycine are reported in table 1. Here in the case of glycine, it has been found both that the MPA and HPA schemes predict $\mathrm{N}_{3}$ attached to $\mathrm{C}_{1}$ has higher $f_{k}^{-}$value for electrophilic attack. The observed electrophilicity trend in all the conformers is $\mathrm{N}_{3}>\mathrm{O}_{5}>\mathrm{O}_{4}$. The observation of the reactive sites by $(s f)_{k}$ is found almost identical to $f_{k}$. Even though the $(s f)_{k}$ values are numerically less it should be worth noting that the values are positive and the ordering of the reactivity has not been changed in any cases. Both the schemes show that $\mathrm{C}_{1}$ and $\mathrm{O}_{5}$ in carboxyl group as the reactive sites for receiving a nucleophile.

In table 2 , the condensed $f_{k}$ and atomic descriptor $(s f)_{k}$ values for the atoms of alanine are shown. As expected, in almost all the cases the $\mathrm{N}$ atom is found to the most preferred site for protonation since in this conformer there is an intra molecular interaction

Table 1. Condensed Fukui function $f_{k}$ and new descriptors $(s f)_{k}\left(10^{-2}\right)$ for glycine.

\begin{tabular}{|c|c|c|c|c|c|c|c|c|}
\hline \multirow[b]{2}{*}{ Atoms } & \multicolumn{4}{|c|}{ B1-DZP/MPA } & \multicolumn{4}{|c|}{ B1-DZP/HPA } \\
\hline & $f_{k}^{+}$ & $f_{k}^{-}$ & $s_{k}^{+} f_{k}^{+}$ & $s_{k}^{-} f_{k}^{-}$ & $f_{k}^{+}$ & $f_{k}^{-}$ & $s_{k}^{+} f_{k}^{+}$ & $s_{k} f_{k}^{-}$ \\
\hline $\mathrm{C}_{1}$ & $0 \cdot 2625$ & $0 \cdot 0987$ & 1.4188 & $0 \cdot 2006$ & $0 \cdot 2261$ & $0 \cdot 0772$ & $0 \cdot 1052$ & $0 \cdot 1227$ \\
\hline $\mathrm{C}_{2}$ & $-0 \cdot 0606$ & $-0 \cdot 0009$ & 0.0756 & $0 \cdot 0000$ & $0 \cdot 0676$ & $0 \cdot 0716$ & $0 \cdot 0940$ & $0 \cdot 1055$ \\
\hline $\mathrm{N}_{3}$ & $0 \cdot 0235$ & $0 \cdot 2248$ & 0.0114 & $1 \cdot 0405$ & $0 \cdot 0499$ & $0 \cdot 2473$ & $0 \cdot 0512$ & $0 \cdot 1259$ \\
\hline $\mathrm{O}_{4}$ & $0 \cdot 0696$ & $0 \cdot 0644$ & 0.0997 & $0 \cdot 0854$ & $0 \cdot 1223$ & $0 \cdot 0970$ & $0 \cdot 3078$ & $0 \cdot 1936$ \\
\hline $\mathrm{O}_{5}$ & $0 \cdot 1835$ & $0 \cdot 1801$ & 0.6933 & 0.6679 & $0 \cdot 2177$ & $0 \cdot 1824$ & 0.9754 & 0.6847 \\
\hline $\mathrm{H}_{6}$ & $0 \cdot 1605$ & $0 \cdot 0913$ & 0.5304 & $0 \cdot 1716$ & $0 \cdot 0915$ & $0 \cdot 0575$ & $0 \cdot 1723$ & $0 \cdot 0680$ \\
\hline $\mathrm{H}_{7}$ & $0 \cdot 1602$ & $0 \cdot 0914$ & 0.5284 & $0 \cdot 1720$ & $0 \cdot 0915$ & 0.0575 & $0 \cdot 1723$ & 0.0680 \\
\hline $\mathrm{H}_{8}$ & $0 \cdot 1095$ & $0 \cdot 0621$ & $0 \cdot 2469$ & $0 \cdot 0794$ & $0 \cdot 0713$ & $0 \cdot 0527$ & $0 \cdot 1046$ & 0.0572 \\
\hline $\mathrm{H}_{9}$ & $0 \cdot 0457$ & $0 \cdot 0941$ & 0.0430 & $0 \cdot 1823$ & 0.0311 & 0.0783 & $0 \cdot 0199$ & $0 \cdot 1262$ \\
\hline $\mathrm{H}_{10}$ & 0.0456 & $0 \cdot 0940$ & $0 \cdot 0428$ & $0 \cdot 1819$ & 0.0311 & 0.0783 & 0.0199 & $0 \cdot 1262$ \\
\hline
\end{tabular}


Table 2. Condensed Fukui function $f_{k}$ and new descriptors $(s f)_{k}\left(10^{-2}\right)$ for alanine.

\begin{tabular}{|c|c|c|c|c|c|c|c|c|}
\hline \multirow[b]{2}{*}{ Atoms } & \multicolumn{4}{|c|}{ B1-DZP/MPA } & \multicolumn{4}{|c|}{ B1-DZP/HPA } \\
\hline & $f_{k}^{+}$ & $f_{k}^{-}$ & $s_{k}^{+} f_{k}^{+}$ & $s_{k}^{-} f_{k}^{-}$ & $f_{k}^{+}$ & $f_{k}^{-}$ & $s_{k}^{+} f_{k}^{+}$ & $\overline{s_{k}} f_{\bar{k}}^{-}$ \\
\hline $\mathrm{C}_{1}$ & $0 \cdot 1734$ & $0 \cdot 0982$ & 0.6248 & $0 \cdot 2004$ & $0 \cdot 1554$ & $0 \cdot 0721$ & $0 \cdot 5016$ & $0 \cdot 1080$ \\
\hline $\mathrm{C}_{2}$ & $-0 \cdot 0568$ & $-0 \cdot 0273$ & $0 \cdot 0067$ & $0 \cdot 0155$ & $0 \cdot 0468$ & $0 \cdot 0536$ & $0 \cdot 0455$ & 0.0597 \\
\hline $\mathrm{N}_{3}$ & $0 \cdot 0031$ & $0 \cdot 2150$ & $0 \cdot 0010$ & $0 \cdot 9605$ & $0 \cdot 0468$ & $0 \cdot 2270$ & $0 \cdot 0455$ & 1.0703 \\
\hline $\mathrm{O}_{4}$ & $0 \cdot 0296$ & 0.0579 & $0 \cdot 0182$ & 0.0697 & $0 \cdot 1030$ & $0 \cdot 0889$ & $0 \cdot 2203$ & $0 \cdot 1641$ \\
\hline $\mathrm{O}_{5}$ & $0 \cdot 1201$ & $0 \cdot 1694$ & $0 \cdot 2997$ & 0.5963 & $0 \cdot 1560$ & $0 \cdot 1708$ & $0 \cdot 5061$ & $0 \cdot 6059$ \\
\hline $\mathrm{H}_{6}$ & $0 \cdot 1666$ & $0 \cdot 0608$ & $0 \cdot 5768$ & $0 \cdot 0768$ & $0 \cdot 0902$ & $0 \cdot 0504$ & $0 \cdot 1690$ & $0 \cdot 0528$ \\
\hline $\mathrm{H}_{7}$ & $0 \cdot 1926$ & $0 \cdot 0858$ & 0.7708 & $0 \cdot 1529$ & 0.0736 & 0.0509 & $0 \cdot 1125$ & 0.0538 \\
\hline $\mathrm{C}_{8}$ & $-0 \cdot 1497$ & -0.0284 & 0.4657 & $0 \cdot 0168$ & 0.0686 & $0 \cdot 0380$ & 0.0977 & 0.0300 \\
\hline $\mathrm{H}_{9}$ & 0.0579 & $0 \cdot 0909$ & 0.0697 & $0 \cdot 1717$ & $0 \cdot 0365$ & 0.0718 & 0.0277 & $0 \cdot 1071$ \\
\hline $\mathrm{H}_{10}$ & $0 \cdot 0728$ & $0 \cdot 0892$ & $0 \cdot 1101$ & $0 \cdot 1653$ & $0 \cdot 0385$ & $0 \cdot 0727$ & 0.0308 & $0 \cdot 1098$ \\
\hline $\mathrm{H}_{11}$ & $0 \cdot 1047$ & $0 \cdot 0528$ & $0 \cdot 2278$ & 0.0579 & 0.0347 & 0.0279 & 0.0575 & $0 \cdot 0162$ \\
\hline $\mathrm{H}_{12}$ & $0 \cdot 0780$ & $0 \cdot 0645$ & $0 \cdot 1264$ & $0 \cdot 0865$ & $0 \cdot 0457$ & $0 \cdot 0347$ & 0.0434 & 0.0250 \\
\hline $\mathrm{H}_{13}$ & $0 \cdot 2077$ & 0.0711 & $0 \cdot 8964$ & $0 \cdot 1051$ & $0 \cdot 0862$ & $0 \cdot 0412$ & $0 \cdot 1543$ & 0.0353 \\
\hline
\end{tabular}

Table 3. Condensed Fukui function $f_{k}$ and new descriptors $(s f)_{k}\left(10^{-2}\right)$ for aniline.

\begin{tabular}{|c|c|c|c|c|c|c|c|c|}
\hline \multirow[b]{2}{*}{ Atoms } & \multicolumn{4}{|c|}{ B1-DZP/MPA } & \multicolumn{4}{|c|}{ B1-DZP/HPA } \\
\hline & $f_{k}^{+}$ & $f_{k}^{-}$ & $s_{k}^{+} f_{k}^{+}$ & $\overline{s_{k}} f_{k}^{-}$ & $f_{k}^{+}$ & $f_{k}^{-}$ & $s_{k}^{+} f_{k}^{+}$ & $\overline{s_{k}} f_{k}^{-}$ \\
\hline $\mathrm{C}_{1}$ & $0 \cdot 1037$ & $0 \cdot 0468$ & $0 \cdot 2608$ & 0.0531 & $0 \cdot 1095$ & $0 \cdot 0769$ & $0 \cdot 2909$ & $0 \cdot 1435$ \\
\hline $\mathrm{C}_{2}$ & $0 \cdot 0340$ & $0 \cdot 1093$ & $0 \cdot 0280$ & $0 \cdot 2897$ & 0.0533 & $0 \cdot 0744$ & 0.0689 & $0 \cdot 1343$ \\
\hline $\mathrm{C}_{3}$ & $0 \cdot 1035$ & $0 \cdot 0471$ & $0 \cdot 2598$ & $0 \cdot 0538$ & $0 \cdot 1097$ & $0 \cdot 0768$ & $0 \cdot 2919$ & $0 \cdot 1431$ \\
\hline $\mathrm{C}_{4}$ & $0 \cdot 1161$ & $0 \cdot 0529$ & $0 \cdot 3269$ & $0 \cdot 0679$ & $0 \cdot 1184$ & 0.0635 & $0 \cdot 3401$ & $0 \cdot 0978$ \\
\hline $\mathrm{C}_{5}$ & $-0 \cdot 0068$ & $0 \cdot 0700$ & $0 \cdot 0011$ & $0 \cdot 1188$ & $0 \cdot 0592$ & $0 \cdot 1240$ & $0 \cdot 0850$ & $0 \cdot 3730$ \\
\hline $\mathrm{C}_{6}$ & $0 \cdot 1163$ & 0.0531 & $0 \cdot 3280$ & $0 \cdot 0684$ & $0 \cdot 1187$ & 0.0634 & $0 \cdot 3418$ & 0.0975 \\
\hline $\mathrm{N}_{7}$ & $0 \cdot 0447$ & $0 \cdot 1512$ & $0 \cdot 0484$ & 0.5544 & 0.0597 & $0 \cdot 1651$ & $0 \cdot 0865$ & 0.6613 \\
\hline $\mathrm{H}_{8}$ & $0 \cdot 0304$ & 0.0548 & $0 \cdot 0224$ & $0 \cdot 0728$ & $0 \cdot 0423$ & 0.0666 & $0 \cdot 0434$ & $0 \cdot 1076$ \\
\hline $\mathrm{H}_{9}$ & 0.0306 & $0 \cdot 0548$ & $0 \cdot 0227$ & $0 \cdot 0728$ & $0 \cdot 0424$ & 0.0666 & $0 \cdot 0436$ & $0 \cdot 1076$ \\
\hline $\mathrm{H}_{10}$ & $0 \cdot 0872$ & $0 \cdot 0660$ & $0 \cdot 1844$ & $0 \cdot 1056$ & 0.0577 & 0.0419 & $0 \cdot 0808$ & $0 \cdot 0426$ \\
\hline $\mathrm{H}_{11}$ & 0.0916 & 0.0683 & $0 \cdot 2035$ & $0 \cdot 1131$ & $0 \cdot 0621$ & 0.0440 & 0.0936 & $0 \cdot 0470$ \\
\hline $\mathrm{H}_{12}$ & 0.0696 & $0 \cdot 0915$ & $0 \cdot 1175$ & $0 \cdot 2030$ & $0 \cdot 0467$ & 0.0515 & $0 \cdot 0529$ & $0 \cdot 0643$ \\
\hline $\mathrm{H}_{13}$ & $0 \cdot 0918$ & 0.0683 & $0 \cdot 2044$ & $0 \cdot 1131$ & 0.0621 & 0.0440 & 0.0936 & $0 \cdot 0470$ \\
\hline $\mathrm{H}_{14}$ & 0.0871 & 0.0659 & $0 \cdot 1840$ & $0 \cdot 1053$ & 0.0578 & $0 \cdot 0418$ & $0 \cdot 0810$ & $0 \cdot 0424$ \\
\hline
\end{tabular}

Table 4. Condensed Fukui function $f_{k}$ and new descriptors $(s f)_{k}\left(10^{-2}\right)$ for $\mathrm{BH}_{2} \mathrm{Cl}$.

\begin{tabular}{|c|c|c|c|c|c|c|c|c|}
\hline \multirow[b]{2}{*}{ Atoms } & \multicolumn{4}{|c|}{ B1-DZP/MPA } & \multicolumn{4}{|c|}{ B1-DZP/HPA } \\
\hline & $f_{k}^{+}$ & $f_{k}^{-}$ & $s_{k}^{+} f_{k}^{+}$ & $s_{k}^{-} f_{k}^{-}$ & $f_{k}^{+}$ & $f_{k}^{-}$ & $s_{k}^{+} f_{k}^{+}$ & $s_{k}^{-} f_{k}^{-}$ \\
\hline $\mathrm{B}_{1}$ & 0.5752 & $0 \cdot 1983$ & $7 \cdot 0968$ & $0 \cdot 8435$ & $0 \cdot 4651$ & $0 \cdot 1914$ & $4 \cdot 6400$ & 0.7858 \\
\hline $\mathrm{Cl}_{2}$ & $0 \cdot 2220$ & 0.5090 & 1.0571 & $5 \cdot 5573$ & $0 \cdot 2832$ & 0.5106 & $1 \cdot 7203$ & $5 \cdot 5923$ \\
\hline $\mathrm{H}_{3}$ & $0 \cdot 1014$ & $0 \cdot 1464$ & $0 \cdot 2205$ & 0.4597 & $0 \cdot 1258$ & $0 \cdot 1490$ & $0 \cdot 3395$ & 0.4762 \\
\hline $\mathrm{H}_{4}$ & $0 \cdot 1014$ & $0 \cdot 1464$ & 0.2205 & 0.4597 & $0 \cdot 1258$ & $0 \cdot 1490$ & $0 \cdot 3395$ & 0.4762 \\
\hline
\end{tabular}

that takes place between the $\mathrm{N}-\mathrm{H}$...O group. The order of nucleophilic site as obtained by HPA is $\mathrm{N}_{3}>\mathrm{O}_{5}>\mathrm{O}_{3}$. The MPA scheme shows that $\mathrm{C}_{1}$ and $\mathrm{H}_{13}$ attached to carbon atom as the most reactive site during nucleophilic attack whereas HPA shows $\mathrm{C}_{1}$ and $\mathrm{O}_{5}$ in carboxyl group as the reactive sites for receiving a nucleophile. Looking deeper into the reactive sites predicted by $f_{k}$ and $(s f)_{k}$ for glycine and 


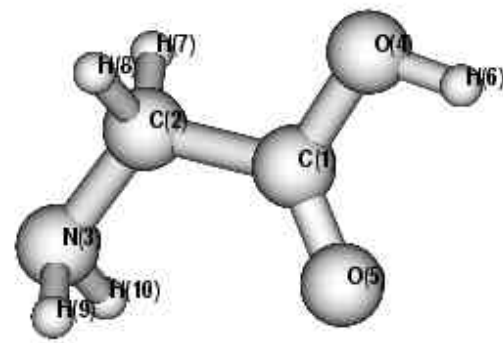

Glycine

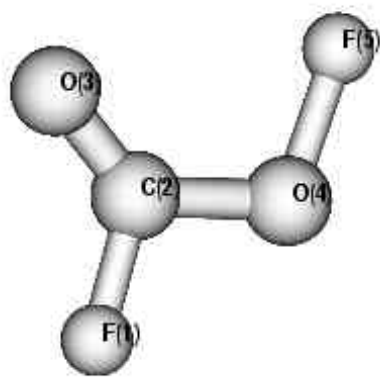

trans $-\mathrm{FC}(\mathrm{O}) \mathrm{OF}$

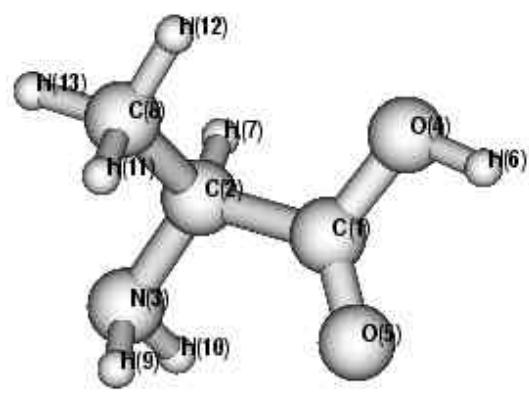

Alanine

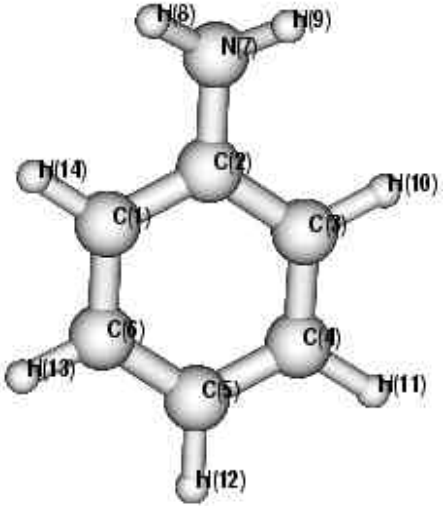

Aniline

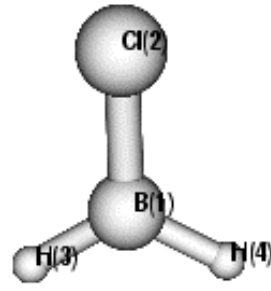

$\mathrm{BH}_{2} \mathrm{Cl}$

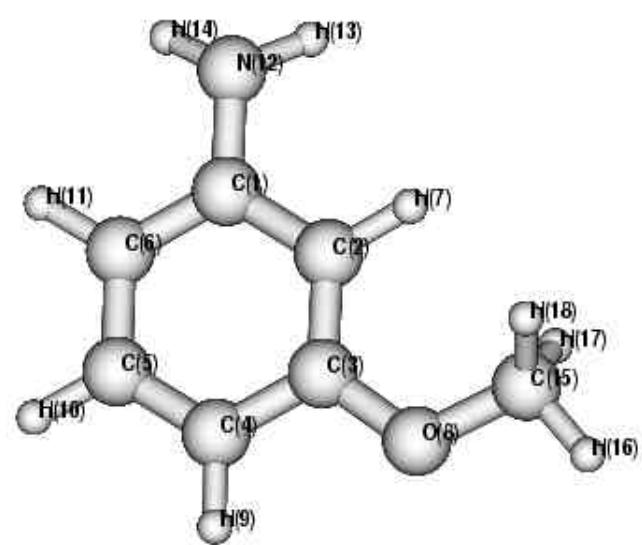

$m$-Anisidine

Figure 1. Equilibrium geometries of molecules taken in the present study (atomic sites are numbered).

Table 5. Condensed Fukui function $f_{k}$ and new descriptors $(s f)_{k}\left(10^{-2}\right)$ for trans-FC(O)OF.

\begin{tabular}{|c|c|c|c|c|c|c|c|c|}
\hline \multirow[b]{2}{*}{ Atoms } & \multicolumn{4}{|c|}{ B1-DZP/MPA } & \multicolumn{4}{|c|}{ B1-DZP/HPA } \\
\hline & $f_{k}^{+}$ & $f_{k}^{-}$ & $s_{k}^{+} f_{k}^{+}$ & $s_{k}^{-} f_{k}^{-}$ & $f_{k}^{+}$ & $f_{k}^{-}$ & $s_{k}^{+} f_{k}^{+}$ & $s_{k} f_{k}^{-}$ \\
\hline $\mathrm{F}_{1}$ & $0 \cdot 0775$ & $0 \cdot 1412$ & $0 \cdot 1598$ & 0.5305 & $0 \cdot 1338$ & $0 \cdot 1640$ & $0 \cdot 4760$ & $0 \cdot 7157$ \\
\hline $\mathrm{C}_{2}$ & $0 \cdot 1855$ & $0 \cdot 1841$ & 0.9157 & 0.9019 & $0 \cdot 3620$ & $0 \cdot 1643$ & $3 \cdot 4871$ & 0.7183 \\
\hline $\mathrm{O}_{3}$ & $0 \cdot 124$ & $0 \cdot 3667$ & 0.4092 & $3 \cdot 5782$ & $0 \cdot 1006$ & $0 \cdot 1347$ & $0 \cdot 2693$ & 0.4828 \\
\hline $\mathrm{O}_{4}$ & $0 \cdot 2816$ & $0 \cdot 1544$ & $2 \cdot 1101$ & 0.6343 & $0 \cdot 0140$ & $0 \cdot 3720$ & 0.5216 & $3 \cdot 6824$ \\
\hline $\mathrm{F}_{5}$ & $0 \cdot 3313$ & $0 \cdot 1537$ & 2.9207 & 0.6286 & $0 \cdot 2636$ & $0 \cdot 1650$ & $1 \cdot 8490$ & $0 \cdot 7245$ \\
\hline
\end{tabular}

alanine, it is clear that in these systems the formation of reactive sites depend mainly on the presence of intramolecular hydrogen bonding. It is worthy of mention that the $(s f)_{k}$ also predict the reactivity as FF values predict, which confirms that these new atomic descriptors can also be used to study the local reactivity in molecular systems.
Analysis on the reliability of newly derived descriptor has been further extended to certain organic molecules. Calculated condensed FF values and the $(s f)_{k}$ values for aniline are given in table 3 . Aniline and its $p$-substituted systems are found to be $\mathrm{N}$-protonated and this was concluded by Summerhays et $a l^{32}$ in their experimental study. Roy et $a l^{19}$ have 
Table 6. Condensed Fukui function $f_{k}$ and new descriptors $(s f)_{k}\left(10^{-2}\right)$ for $m$-anisidine.

\begin{tabular}{|c|c|c|c|c|c|c|c|c|}
\hline \multirow[b]{2}{*}{ Atoms } & \multicolumn{4}{|c|}{ B1-DZP/MPA } & \multicolumn{4}{|c|}{ B1-DZP/HPA } \\
\hline & $f_{k}^{+}$ & $f_{k}^{-}$ & $s_{k}^{+} f_{k}^{+}$ & $s_{k}^{-} f_{k}^{-}$ & $f_{k}^{+}$ & $f_{k}^{-}$ & $s_{k}^{+} f_{k}^{+}$ & $s_{k}^{-} f_{k}^{-}$ \\
\hline $\mathrm{C}_{4}$ & $0 \cdot 0628$ & $0 \cdot 0808$ & $0 \cdot 0942$ & $0 \cdot 1559$ & $0 \cdot 0796$ & $0 \cdot 1234$ & $0 \cdot 1513$ & $0 \cdot 3636$ \\
\hline $\mathrm{C}_{6}$ & $0 \cdot 0343$ & $0 \cdot 0681$ & $0 \cdot 0281$ & $0 \cdot 1107$ & 0.0575 & $0 \cdot 1104$ & $0 \cdot 0790$ & $0 \cdot 2911$ \\
\hline $\mathrm{N}_{12}$ & $0 \cdot 0012$ & $0 \cdot 0781$ & $0 \cdot 0000$ & $0 \cdot 1457$ & $0 \cdot 0627$ & $0 \cdot 1153$ & $0 \cdot 0939$ & $0 \cdot 3175$ \\
\hline
\end{tabular}

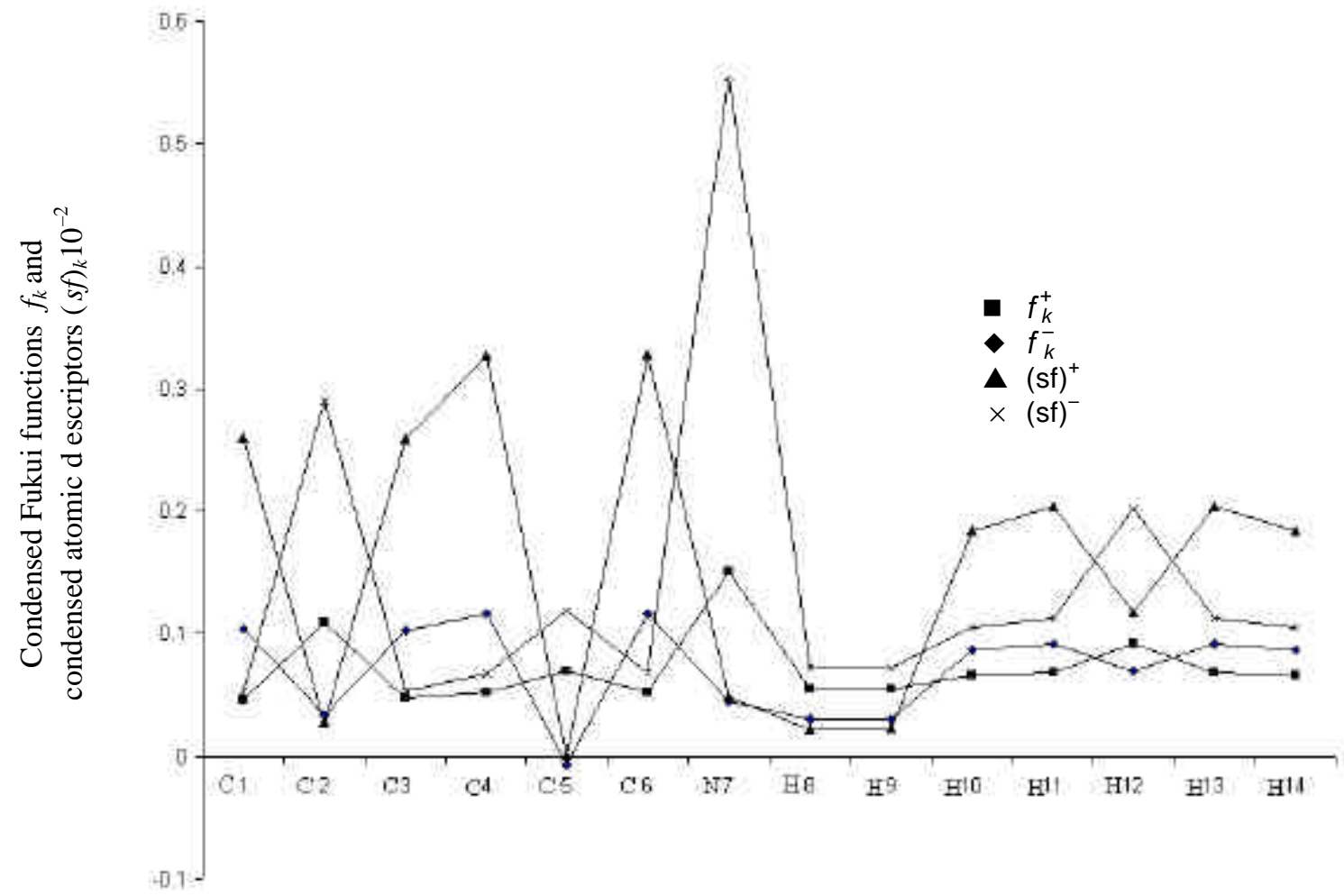

Figure 2. Condensed Fukui function and condensed atomic descriptors of aniline by MPA gross charges using B1DZP level of theory.

proved theoretically that $\mathrm{N}$ is the preferred site for protonation also. It is clear from the values of FF and $(s f)_{k}$ that $\mathrm{N}$ is a possible site for protonation and the results are in agreement with previous studies. In the case of nucleophilic attack, the atoms $\mathrm{C}_{4}$ and $\mathrm{C}_{6}$ are found to be the most preferred sites. The graphical representation of condensed Fukui function and condensed atomic descriptors calculated by MPA charges is given in figure 2 . It clearly shows that the condensed atomic descriptors predict the reactive sites as that of the condensed Fukui functions without disturbing the trend. Arulmozhiraja $e t a l^{33}$ have made a detailed study on the reactive sites of several organic molecules and some isomers based on atomic charges calculated by MPA and NPA analysis at the local sites of a molecule. We have chosen $\mathrm{BH}_{2} \mathrm{Cl}$ and trans- $\mathrm{FC}(\mathrm{O}) \mathrm{OF}$ molecules to discuss the reliability of atomic descriptors. In the case of $\mathrm{BH}_{2} \mathrm{Cl}, \mathrm{FF}$ and $(s f)_{k}$ values using different population schemes are depicted in table 4 . The reactive sites evaluated ${ }^{33}$ are as follows: $f^{+}, \mathrm{B}>\mathrm{Cl}>\mathrm{H} ; f^{-}$, $\mathrm{Cl}>\mathrm{H}>\mathrm{B}$. The order of reactive sites calculated in the present study matches well with the order predicted by the previous work. The calculated $f_{k}$ values predict that $\mathrm{B}$ and $\mathrm{Cl}$ atoms are the possible sites for nucleophilic and electrophilic attacks, respectively. The atomic descriptors $(s f)_{k}$ also predicts the same reactivity with the same order and the values are found to be positive. For trans-FC(O)OF system the calculated $\mathrm{FF}$ and $(s f)_{k}$ values are given in table 5. The calculated condensed $\mathrm{FF}$ values thus predict $\mathrm{F}_{5}$ attached to $\mathrm{O}_{4}$ and $\mathrm{O}_{4}$ atom attached to $\mathrm{F}_{5}$ as possible reactive sites for nucleophilic and electrophilic attacks respectively. Hence the reactive sites and the 
order of reactivity produced by the new atomic descriptor are found to be identical with that of $f_{k}$ values and the values are positive. In order to discuss the importance of the atomic descriptor, the protonation site of the molecule $m$-anisidine has also been considered in this study. We have given only the most favourable reactive sites in the table 6 . Both the MPA and HPA schemes predict that $\mathrm{C}_{4}$ atom to be the most reactive site for protonation during electrophilic attack, as reported by Summerhays et $a l^{32}$ experimentally.

\section{Conclusion}

In this paper, we have introduced an atomic descriptor $(s f)_{k}$ to determine the local reactive sites for the molecular systems during electrophilic, nucleophilic and radical attacks. The results clearly show once again the superiority of HPA over MPA scheme. An analysis on the nature of newly derived "atomic descriptors" demonstrates that the issue of negative Fukui functions has been overcome. The reactive sites predicted by condensed Fukui functions $f_{k}$ during electrophilic, nucleophilic and radical attacks are successfully reproduced by these new condensed atomic descriptors $(s f)_{k}$. This descriptor is found to be influential, since, it contains information on both the local reactivity and selectivity of a molecule. Even though the ordering of reactive sites in the molecules has not changed, the numerical values of the descriptors are found to be very low. This quantity is always positive and shows reactive trends as Fukui function predicts.

\section{References}

1. Hohenberg P and Kohn W 1964 Phys. Rev. B136 864

2. Parr R G, Donnelly R A, Levy M and Palke W E J 1978 Chem. Phys. 683801

3. Perdew J P, Parr R G, Levy M and Balduz J L 1982 Phys. Rev. Lett. 49 1691; Pal S, Roy R K and Chandra 1994 J. Phys. Chem. 982314

4. Gosh S K and Berkowitz M 1985 J. Chem. Phys. 83 2976

5. Yang W and Parr R G 1985 Proc. Natl. Acad. Sci. USA 826723

6. Geerlings $\mathrm{P}$, Boon $\mathrm{G}$, van Alsenoy $\mathrm{C}$ and De Proft $\mathrm{F}$ 2005 Int. J. Quantum Chem. 101722

7. Langenaekar W, De Proft F and Geerlings P $1995 \mathrm{~J}$. Phys. Chem. 996424

8. Roy R K, Krishnamurti S, Geerlings $\mathrm{P}$ and Pal S 1998 J. Phys. Chem. 1023746
9. De Proft F, Langenaekar W and Geerlings P 1993 J. Phys. Chem. 97 1876; Langenaekar W, Coussement N, De Proft F and Geerlings P 1994 J. Phys. Chem. 98 3010; De Proft F, Amira S, Choho K and Geerlings P 1994 J. Phys. Chem. 98 5227; Langenaekar W, Demel K and Geerlings P 1992 J. Mol. Struct: Theochem. 259 317; Langenaekar W, Demel K and Geerlings P 1991 J. Mol. Struct: Theochem. 234 329; Baeten A, De Proft F, Langenaekar W and Geerlings P 1994 J. Mol. Struct: Theochem. 306 203; Chandra A K, Geerlings P and Nguyen M T 1997 J. Org. Chem. 62 6417; Sengupta D, Chandra A K and Nguyen M T 1997 J. Org. Chem. 626404

10. Krishnamurti S, Roy R K, Vetrivel R, Iwata S and Pal S 1997 J. Phys. Chem. 1017253

11. Parr R G and Yang W J 1984 Am. Chem. Soc. 1064049

12. Yang W and Mortier W J 1986 J. Am. Chem. Soc. 1085708

13. Nalewajski R F 1993 Structure and bonding (Berlin: Springer) vol. 80, p. 115

14. Cioslowski J, Martinov M and Mixon S T 1993 J. Phys. Chem. 9710948

15. Bader R W F 1990 Atoms in molecules a quantum theory, Oxford Science Publications (London: Clarendon)

16. Komorowski L, Lipinski J and Pyka M J 1993 J. Phys. Chem. 97 3166; Komorowski L 1993 Structure and bonding (Berlin: Springer) vol. 80, p. 46

17. Senthilkumar L and Kolandaivel P 2005 Mol. Phys. 103547

18. Roy R K, Hirao K, Krishnamurty S and Pal S J 2001 Chem. Phys. 115 2901; Bultinck P, Carbó-Dorca R and Langenaeker W J 2003 Chem. Phys. 118 4349; Bultinck P and Carbó-Dorca R J 2003 Math. Chem. 3467

19. Roy R K, Pal S and Hirao K 1999 J. Chem. Phys. 110 8236

20. Roy R K, Hirao K and Pal S 2000 J. Chem. Phys. 113 1372

21. Mulliken R S 1955 J. Chem. Phys. 231833

22. Reed A E and Weinhold F 1983 J. Chem. Phys. F8 4066

23. Hirshfeld F L 1977 Theor. Chim. Acta 44129

24. Chattaraj P K, Maiti B and Sarkar U 2003 J. Phys. Chem. A107 4873

25. Császár A G 1992 J. Am. Chem. Soc. 1149568

26. Császár A G 1996 J. Phys. Chem. 1003541

27. De Proft F, Jan Martin M L and Geerlings P 1996 Chem. Phys. Lett. 256400

28. Vosko S H, Wilk L and Nusair M 1980 Can. J. Phys. 581200

29. Huzinaga S 1965 J. Chem. Phys. 421293

30. Baerends E J et al 2004 Amsterdam Density Functional Package, ADF 2004.01

31. Pérez P, Parra-Mouchet J and Contreras R R $2004 J$. Chilean Chem. Soc. 49

32. Summerhays K D, Pollack S K, Taft R W and Hehre W J 1977 J. Am. Chem. Soc. 999585

33. Arulmozhiraja S and Kolandaivel P 1997 Mol. Phys. 9055 